\title{
Myricetin against ischemic cerebral injury in rat middle cerebral artery occlusion model
}

\author{
LONG SUN $^{1 *}$, PENG XU $^{1 *}$, TINGGANG FU $^{1}, \mathrm{XIN} \mathrm{HUANG}^{1}, \mathrm{JIE} \mathrm{SONG}^{1}$, \\ MENG CHEN $^{2}$, XINGHAN TIAN ${ }^{2}$, HONGLI YIN ${ }^{3}$ and JICHUN HAN ${ }^{3}$ \\ ${ }^{1}$ Department of Neurosurgery, Lin Yi Central Hospital, Linyi, Shandong 276400; \\ ${ }^{2}$ Department of Medicine, Yantai Yuhuangding Hospital of Laishan Branch, Yantai, Shandong 264003; \\ ${ }^{3}$ State Key Laboratory of Natural Medicines, China Pharmaceutical University, Nanjing, Jiangsu 210009, P.R. China
}

Received July 15, 2017; Accepted November 9, 2017

DOI: $10.3892 / \mathrm{mmr} .2017 .8212$

\begin{abstract}
The purpose of the present study was to examine the effects of myricetin on reducing cerebral ischemia injury in a rat model. A rat model of permanent middle cerebral artery occlusion (pMCAO) was used in the present study. Rats were randomized into the following five groups: Sham, model, low-myricetin $(1 \mathrm{mg} / \mathrm{kg})$, medium-myricetin $(5 \mathrm{mg} / \mathrm{kg})$ and high-myricetin (25 mg/kg) groups. Neurological deficit scores were evaluated by an examiner blinded to the experimental groups. Brain infarct size was estimated macroscopically using 2,3,5-triphenyltetrazolium chloride staining. The levels of inflammatory factors tumor necrosis factor (TNF)- $\alpha$, interleukin (IL)- 6 and IL-1 $\beta$, and oxidative stress index superoxide dismutase (SOD), malondiadehyde (MDA), and the glutathione/glutathione disulfide (GSH/GSSG) ratio were measured by ELISA. The degree of brain cell apoptosis was determined using a terminal deoxynucleotidyl transferase dUTP nick-end labeling assay. Protein expression levels of total or phosphorylated p38 mitogen activated protein kinase (MAPK), nuclear factor (NF)- $\mathrm{kB} / \mathrm{p} 65$ and protein kinase $\mathrm{B}$ (AKT) were determined using a western blotting assay. The neurological deficit score and infarct area induced by pMCAO decreased in a dose-dependent manner following myricetin treatment. Furthermore, myricetin reduced the expression levels of IL-1 $\beta$, IL- 6 , TNF- $\alpha$, and MDA, and increased GSH/GSSG ratio and SOD activity. A significant decrease in cell apoptosis was observed in response to myricetin. In addition, myricetin significantly increased the level of phosphorylated AKT protein, and decreased the phosphorylation of p38 MAPK and the level of $\mathrm{NF}-\kappa \mathrm{B} / \mathrm{p} 65$. Overall, the
\end{abstract}

Correspondence to: Dr Long Sun, Department of Neurosurgery, Lin Yi Central Hospital, 17 Jiankang Road, Linyi, Shandong 276400, P.R. China

E-mail: sunlongsun@163.com

*Contributed equally

Key words: cerebral injury, myricetin, anti-apoptosis, anti-inflammation, antioxidant results of the present study suggested that myricetin exhibits a therapeutic effect by reducing ischemic cerebral injury, and the protective effect of myricetin may be associated with the p38 MAPK, NF- $\kappa \mathrm{B} / \mathrm{p} 65$ and AKT signaling pathways.

\section{Introduction}

Stroke is the loss of brain function due to a disturbance in the blood supply to the brain, also referred to as a cerebrovascular accident, or, 'brain attack' $(1,2)$. Stroke is the second leading cause of mortality worldwide, and another primary manifestation of stroke is disability. According to statistics, up to $30 \%$ of stroke survivors are permanently disabled, significantly influencing quality of life and safety (3-6). Therefore, the search for novel ways of treating and preventing stroke is of primary concern.

Of all types of stroke, cerebral ischemic injury is of great importance, however its pathophysiological process is complex. Of all types of pathophysiology in neuronal cells, enhanced extent of neuronal apoptosis and levels of reactive oxygen species (ROS) are considered very significant (7). In addition, inflammation is important in the course of cerebral ischemia injury (8). Studies have demonstrated that cerebral ischemia involves an increase in the release of proinflammatory cytokines [interleukin (IL)-6, IL-1 $\beta$ and tumor necrosis factor (TNF)- $\alpha$, leading to aggravated brain injury (9). The modulation of anti-oxidation, anti-inflammation, and anti-apoptosis is considered a crucial and effective therapeutic strategy in the treatment of cerebral ischemia injury.

Myricetin (3,30,40,5,50,7-hexahydroxyflavone) is a natural herbal flavonoid that exists abundantly in various plant sources, including medicinal herbs, vegetables, fruits, berries and tea leaves. Myricetin has diverse pharmacological activities, including anti-apoptotic function, anti-inflammatory effects, and antioxidant properties (10-12). Studies have revealed that myricetin acts against ischemia/reperfusion-induced acute injury through the activation of cyclooxygenase-2, cytochrome P450 and p38 mitogen-activated protein kinase (p38 MAPK) signaling pathways, and the inhibition of fatty acid synthase and 6-phosphogluconate dehydrogenase signaling pathways in the isolated heart of rats (13). Studies have additionally demonstrated that myricetin attenuates brain injury in a rat 
model of cerebral ischemia via activation of the nuclear factor, erythroid 2 like 2 pathway and reducing oxidative stress (14). Despite these research advances, the anti-inflammatory effect of myricetin on ischemic cerebral injury remains unclear. Therefore, the aim of the present study was to investigate whether myricetin could prevent cerebral ischemic injury and to identify the potential mechanisms involved.

\section{Materials and methods}

Test compounds, chemicals and reagents. Myricetin (purity $\geq 98 \%$ ) was purchased from Chengdu Technology and Market Co., Ltd., (Chengdu, China). Rat superoxide dismutase (SOD), rat malondiadehyde (MDA), rat glutathione (GSH), rat glutathione disulfide (GSSG), rat IL-1 $\beta$, rat IL-6 and rat TNF- $\alpha$ kits was purchased from Enzyme-linked Biotechnology Co., Ltd. (Shanghai, China). In Situ Cell Death Detection kit, POD was purchased from Roche Diagnostics GmbH (Mannheim, Germany). Anti-protein kinase B (AKT) antibody produced in rabbit, anti-phospho-AKT $\left(\mathrm{pSer}^{129}\right)$ antibody produced in rabbit, anti-nuclear factor (NF)- $\mathrm{B}$ p65 antibody produced in rabbit, anti-phospho-NF-кB p65 (pSer281) antibody produced in rabbit, anti-p38 MAPK antibody produced in rabbit, and anti-p38 MAPK phospho (pT180/pY182) antibody produced in rabbit were obtained from Sigma-Aldrich (Merck KGaA, Darmstadt, Germany). 2,3,5-triphenyltetrazolium chloride staining (TTC) and the other chemicals and reagents were purchased from Sigma-Aldrich (Merck KGaA).

Animals. Adult Sprague-Dawley rats (230-280 g) were purchased from Jinan Pengyue Experimental Animal Breeding Co. Ltd. (Zhangqiu, China: http://www.cndss. net/company-jinanpengyueshiyandongwufanyu923.html). All experimental procedures were approved by the Institutional Animal Care and Use Committee of the National Institute Pharmaceutical Education and Research (Linyi, China).

Cerebral ischemia model. Transient focal cerebral ischemia was induced in the rats via the left middle cerebral artery (MCA) occlusion technique, as previously described $(7,9)$. The experiments were performed as follows: Rats were anesthetized by intraperitoneal injection of $10 \%$ chloral hydrate, following a ventral midline neck incision, the right common carotid artery (CCA), the external carotid artery, and the internal carotid artery (ICA) were carefully exposed and dissected away from adjacent nerves. Microvascular aneurysm clips were applied to the right CCA and the ICA. A filament rounded by gentle heating near a flame was introduced through a small incision into the CCA and then into the ICA. The suture was inserted $18-20 \mathrm{~mm}$ from the carotid bifurcation, which was verified by mild resistance, to occlude the MCA. The sham-operated rats underwent the same procedure, except that the filament was not inserted. During the surgery, body temperature was kept at $37^{\circ} \mathrm{C}$ with a heating pad and a warm light. Following incision closure, the rats were returned to standard housing conditions and allowed free access to food and water.

Drug treatment and surgical operation. The 48 rats were randomly divided into five groups for administration of myricetin by oral gavage: Sham group $(n=12)$, model group $(\mathrm{n}=12)$, low (L)-myricetin group (1 mg/kg myricetin + model, $\mathrm{n}=6$ ), medium (M)-myricetin group (5 $\mathrm{mg} / \mathrm{kg}$ myricetin + model, $\mathrm{n}=6)$, and high $(\mathrm{H})$-myricetin group $(25 \mathrm{mg} / \mathrm{kg}$ myricetin + model, $n=12$ ). The sham group received normal saline intra-gastrically (i.g.) once per day for one week, and the sham-operation (the sham-operated rats underwent the same procedure, except that the filament was not inserted) was performed $1 \mathrm{~h}$ following the last administration of normal saline. The model group received normal saline i.g. once per day for one week, and the cerebral ischemia model operation was performed $1 \mathrm{~h}$ following the last administration of normal saline. The three myricetin groups received myricetin i.g. once per day for one week, and the operation was performed $1 \mathrm{~h}$ following the last administration of myricetin. Note: TTC staining required the whole brain, so the brain tissue could not be tested for other indicators (except for evaluation of neurological deficit) following TTC staining. TTC staining and evaluation of neurological deficit occurred only in the L-myricetin and M-myricetin groups, so the number of animals in these two groups was 6 . However, in addition to the TTC staining and evaluation of neurological deficit, there were other tests [including terminal deoxynucleotidyl transferase dUTP nick-end labeling (TUNEL) staining, western blot assay, and assay of oxidative stress and inflammatory factors] in the other three groups (sham, model and H-myricetin groups), therefore the number of animals in these three groups was 12 .

Evaluation of neurological deficit. Following 1 day (24 h) of ischemia, evaluation of the neurological deficit scores was performed by an examiner blinded to the experimental groups. Neurological findings were scored on a five-point scale: No neurological deficits $=0$; failure to extend the right forepaw fully $=1$; circling to the right $=2$; falling to the right $=3$; failure to walk spontaneously with depressed levels of consciousness $=4$. The greater the neurological deficit score, the more severe the impairment (15). A total of 5 groups (sham group, model group, L-myricetin group, M-myricetin group and H-myricetin group) were used for the analysis of neurological deficit.

Determination of cerebral infarct areas. The rats were euthanized $24 \mathrm{~h}$ following permanent middle cerebral artery occlusion (pMCAO). The brains (whole brain) were quickly removed, sliced, fixed, stained, and photographed as described previously (9). The specific experimental operations were as follows: Rats were sacrificed and brains were quickly removed and placed at $-20^{\circ} \mathrm{C}$ for $5 \mathrm{~min}$. Following $5 \mathrm{~min}$ of freezing, the brains were sliced into three $2 \mathrm{~mm}$-thick coronal sections. The sections were incubated in $1 \%$ TTC at $37^{\circ} \mathrm{C}$ for $30 \mathrm{~min}$, and the sections were turned over in $15 \mathrm{~min}$. Normal brain tissues were dyed bright red, and the unstained area (white area) was defined as the ischemic lesion. Infarct areas were measured using Photoshop CS6 image analysis software (Adobe Systems Europe, Ltd., Maidenhead, UK). To compensate for brain edema, the corrected volume was calculated as follows: Percentage hemisphere lesion volume $(\% \mathrm{HLV})=[$ total infarct volume-(left hemisphere volume-right hemisphere volume)]/right hemisphere volume x $100 \%$.

Assay of oxidative stress and inflammatory factors. The brains were harvested $24 \mathrm{~h}$ following the operation and stored 
at $-70^{\circ} \mathrm{C}$ for later analysis. The homogenates extracted from the cortex of the ischemic brain hemispheres were analyzed for biochemical parameters. According to the manufacturers' protocol, the cortexes of the ischemic brain hemispheres were homogenized in $10 \%$ homogenate solution, and the supernatant was collected for biochemical marker analyses.

The levels of SOD activity, MDA, GSG/GSSH ratio, IL-1 $\beta$, IL- 6 and TNF- $\alpha$ levels in brain tissue were determined using a rat SOD kit (cat. no. ml540172), rat malondialdehyde (MDA) kit (cat. no. m1016824), rat T-GSH/HSSG kit (cat. no. m1022385), rat interleukin $1 \beta$ (IL-1 $\beta$ a) kit (cat. no. ml037361), rat interleukin 6 (IL-6) kit (cat. no. ml102828) and rat TNF- $\alpha$ kit (cat. no. m1002859) following the manufacturer's protocol (Enzyme-linked Biotechnology Co., Ltd.). Briefly, the standards and sample diluents were added and incubated for $30 \mathrm{~min}$ at $37^{\circ} \mathrm{C}$, followed by washing 5 times, and then horseradish peroxidase-conjugate reagent was added and incubated for $30 \mathrm{~min}$ at $37^{\circ} \mathrm{C}$. The samples were washed 5 times, and Chromogen solutions A and B were added, incubated for another $30 \mathrm{~min}$ at $37^{\circ} \mathrm{C}$, and then the stop solution was added and the absorbance was measured at the corresponding wavelength ranges within $15 \mathrm{~min}$, and calculated.

TUNEL. The degree of brain cell apoptosis was determined using TUNEL assay as previously described (9). Briefly, the brain tissue was fixed with $10 \%$ neutral-buffered formalin for $48 \mathrm{~h}$ at room temperature (about $25^{\circ} \mathrm{C}$ ). Then the brain tissue was embedded in paraffin (cat. no. YA0015; Beijing Solarbio Science and Technology Co., Ltd., Beijing, China), following deparaffinization and gradient ethanol rehydration the sections were treated with proteinase $\mathrm{K}(10 \mathrm{mM})$ for $15 \mathrm{~min}$ at $37^{\circ} \mathrm{C}$, and the slides were immersed in the TUNEL reaction mixture for $60 \mathrm{~min}$ at $37^{\circ} \mathrm{C}$ in a humidified atmosphere in the dark. Next, the slides were incubated in a converter-POD (50 $\mu \mathrm{l})$ for $30 \mathrm{~min}$ at $37^{\circ} \mathrm{C}$, resulting in the characteristic blue nuclear staining, and were then analyzed using an Eclipse Ti2 inverted microscope (Nikon Corporation, Tokyo, Japan). To evaluate the apoptosis index of TUNEL-stained brain tissues, the TUNEL index (\%) was calculated as the ratio of the number of TUNEL-positive cells divided by the total number of cells. For each sample, the average was calculated from the counting of eight randomly selected areas of TUNEL-stained slices at x400 magnification.

Determination of protein levels via western blotting assay. The frozen brain tissues (cortex) were ground with a mortar and pestle, and then were homogenized with total protein extraction lysis buffer (Beijing Solarbio Science and Technology Co., Ltd). The protein concentration was determined using a Bio-Rad protein assay (Bio-Rad Laboratories Inc., Hercules, CA, USA). The lysates $(30 \mu \mathrm{g})$ were separated by electrophoresis on $10 \%$ sodium dodecyl sulfate-polyacrylamide gels (SDS-PAGE), then transferred to nitrocellulose membranes (GE Healthcare, Chicago, IL, USA), and blocked at room temperature for $2 \mathrm{~h}$ with 5\% non-fat milk in Tris-buffered saline with Tween (TBST). Membranes were incubated with an antibody against rabbit anti-AKT (cat. no. SAB4300574), rabbit anti-phospho-AKT (pSer ${ }^{129}$; cat. no. SAB4300259), rabbit anti-NF- $\mathrm{BB}$ p65 (cat. no. SAB4502610), rabbit anti-phospho-NF-кB p65 (pSer281; Cat. no. SAB4301496), rabbit anti-p38 MAPK (cat. no. SAB4500491) and rabbit anti-p38 MAPK phospho (pT180/pY182; cat. no. SAB5600057) all at dilution: 1:10,000 in 5\% milk/TBST at $4^{\circ} \mathrm{C}$ overnight $(15 \mathrm{~h})$. The membrane was then washed with TBST and incubated with horseradish peroxidase-conjugated antibody (mouse anti-rabbit IgG cat. no. 93702; 1:5,000; Cell Signaling Technology Inc., Danvers, MA, USA) at room temperature $\left(25^{\circ} \mathrm{C}\right)$ for $1 \mathrm{~h}$. The blots were developed with an enhanced chemiluminescence detection kit (Thermo Fisher Scientific, Inc., Waltham, MA, USA) and were exposed on Kodak radiographic film. The immunoblots were visualized with an EC3 Imaging System, and analyzed with VisionWorks ${ }^{\circledR}$ LS Analysis Software (UVP, LLC, Phoenix, AZ, USA).

Ethics statement. All experimental protocols were approved by the Institutional Animal Care and Use Committee of Lin Yi Central Hospital (Linyi, China). The methods were carried out in accordance with the approved guidelines.

Statistical analysis. Data are presented as the mean \pm standard deviation from at least six independent experiments. Statistical differences were determined using analysis of variance followed by Bonferroni correction. The analyses were performed using SPSS version 22.0 software (IBM SPSS, Armonk, NY, USA). $\mathrm{P}<0.05$ was considered to indicate a statistically significant difference.

\section{Results}

Myricetin decreases brain infarct area and neurological deficit scores. The area of brain infarction was measured with TTC staining. As presented in Fig. 1A and B, no infarct area was observed in the sham group. When compared with the sham group, the model group demonstrated a marked increase in brain infarct area $(\mathrm{P}<0.01)$. Pretreatment with M-myricetin and $\mathrm{H}$-myricetin significantly decreased the brain infarct size induced by ischemia $(\mathrm{P}<0.05$ and $\mathrm{P}<0.01)$. As presented in Fig. $1 \mathrm{C}$, pMCAO resulted in a significant increase in the neurological deficit score of the model group $(\mathrm{P}<0.01)$, and a significant decrease was then observed in the M-myricetin and H-myricetin groups $(\mathrm{P}<0.05$ and $\mathrm{P}<0.01)$. The results indicated that myricetin protected against ischemic brain injury, and that the neuroprotective effect of $\mathrm{H}$-myricetin was increased compared with M-myricetin. Considering the significant neuroprotective effects observed with myricetin at $25 \mathrm{mg} / \mathrm{kg}$ body weight (H-myricetin), this concentration was used for the subsequent studies.

Myricetin treatment alleviates oxidative stress in ischemic brain tissue. Previous studies have indicated that oxidative stress results in ischemic cerebral injury. Therefore, the GSH/GSSG ratio, MDA contents, and the SOD activity in the brain tissue were detected. As presented in Fig. 2, compared with sham group, the MDA content increased significantly, whereas the SOD activity and GSH/GSSG ratio decreased significantly in the model group $(\mathrm{P}<0.01)$. However, treatment with $\mathrm{H}$-myricetin significantly reversed these alterations $(\mathrm{P}<0.01)$. Decreased MDA content and increased GSH/GSSG ratio and SOD activity were observed following treatment with H-myricetin. 
A

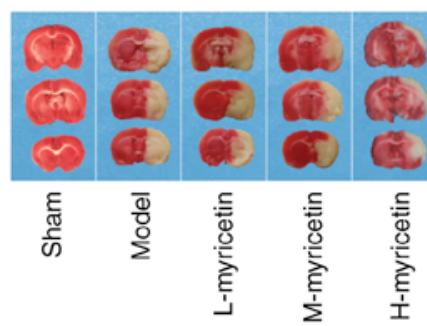

B

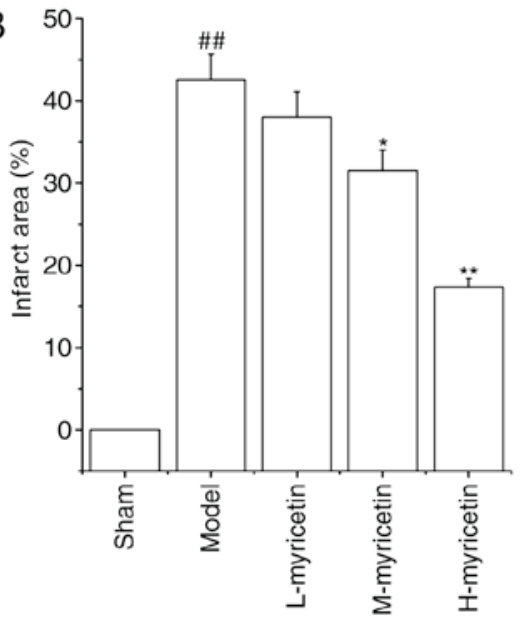

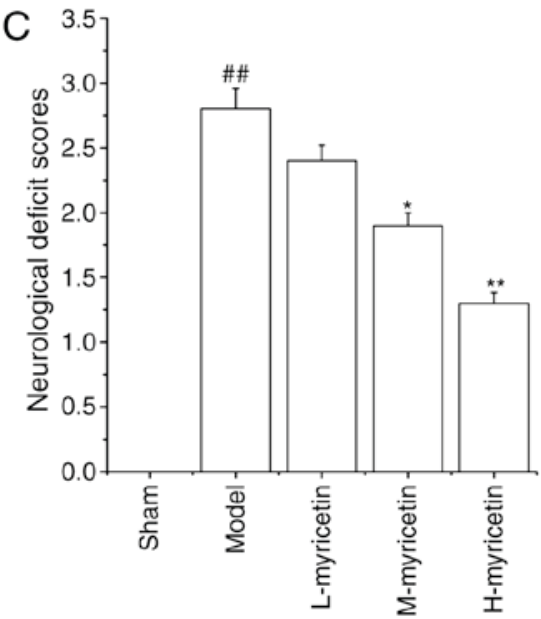

Figure 1. Effects of myricetin on neurological deficits and brain infarct volume. (A) 2,3,5-triphenyltetrazolium chloride-stained sections, the red region indicates the non-ischemic portion of brain, and the pale-colored region indicates the ischemic portion. (B) Evaluation of the infarction area using Photoshop image analysis software. (C) Neuroprotective effects of myricetin based on neurological deficit scores in permanent middle cerebral artery occlusion rats. Data are expressed as the mean \pm standard deviation. ${ }^{\# \#} \mathrm{P}<0.01$ vs. Sham group; ${ }^{*} \mathrm{P}<0.05,{ }^{* *} \mathrm{P}<0.01$ vs. Model group, $\mathrm{n}=6 . \mathrm{L}$, low; $\mathrm{M}$, medium; $\mathrm{H}$, high .
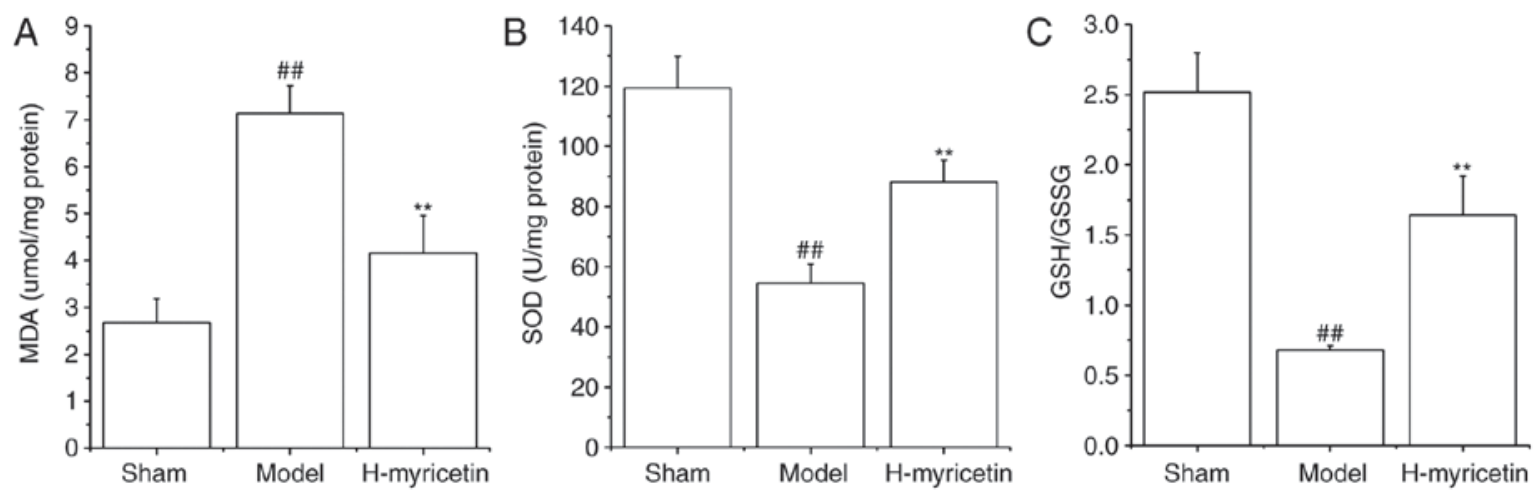

Figure 2. Myricetin preconditioning significantly suppresses oxidative stress. The effect of myricetin on (A) MDA level, (B) SOD activity, and (C) GSH/GSSH ratio in the rat model. Data are expressed as the mean \pm standard deviation. ${ }^{\# \#} \mathrm{P}<0.01$ vs. Sham group; ${ }^{*} \mathrm{P}<0.05,{ }^{* *} \mathrm{P}<0.01$ vs. Model group, $\mathrm{n}=6$. H, high; $\mathrm{SOD}$, superoxide dismutase; MDA, malondiadehyde; GSH/GSSG, glutathione/glutathione disulfide.
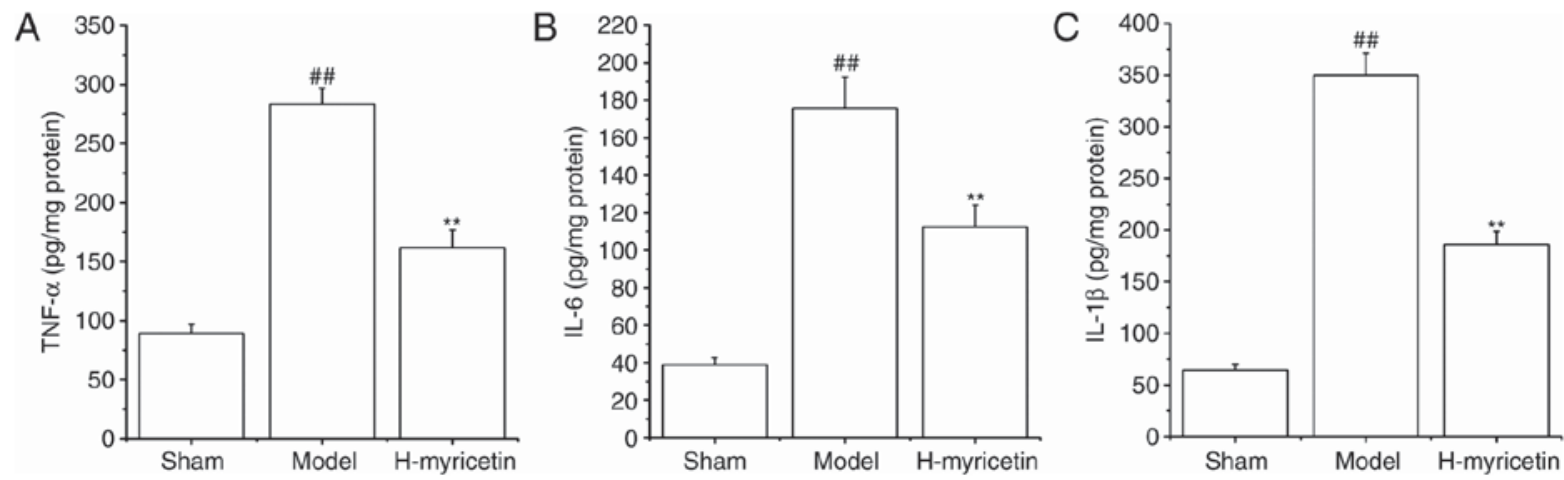

Figure 3. Myricetin preconditioning significantly reduces inflammatory cytokine release. The effect of myricetin on (A) TNF- $\alpha$, (B) IL-6, and (C) IL-1 $\beta$ levels in the rat model. Data are expressed as the mean \pm standard deviation, ${ }^{\# \#} \mathrm{P}<0.01$ vs. Sham group; ${ }^{* *} \mathrm{P}<0.01$ vs. Model group, $\mathrm{n}=6$. H, high; TNF- $\alpha$, tumor necrosis factor- $\alpha$; IL, interleukin.

Myricetin treatment alleviates inflammation in ischemic brain tissue. It has previously been demonstrated that the inflammatory response is critical in ischemic cerebral injury, and that myricetin has an anti-inflammatory effect (13), therefore the present study detected the effects of myricetin on levels of inflammatory cytokines (IL-1 $\beta$, TNF- $\alpha$ and IL-6) in brain tissue. As presented in Fig. 3, compared with the sham group, the levels of IL- $1 \beta$, IL- 6 and TNF- $\alpha$ increased significantly in the model group $(\mathrm{P}<0.01)$. Treatment with $\mathrm{H}$-myricetin significantly reversed these alterations in levels of inflammatory 

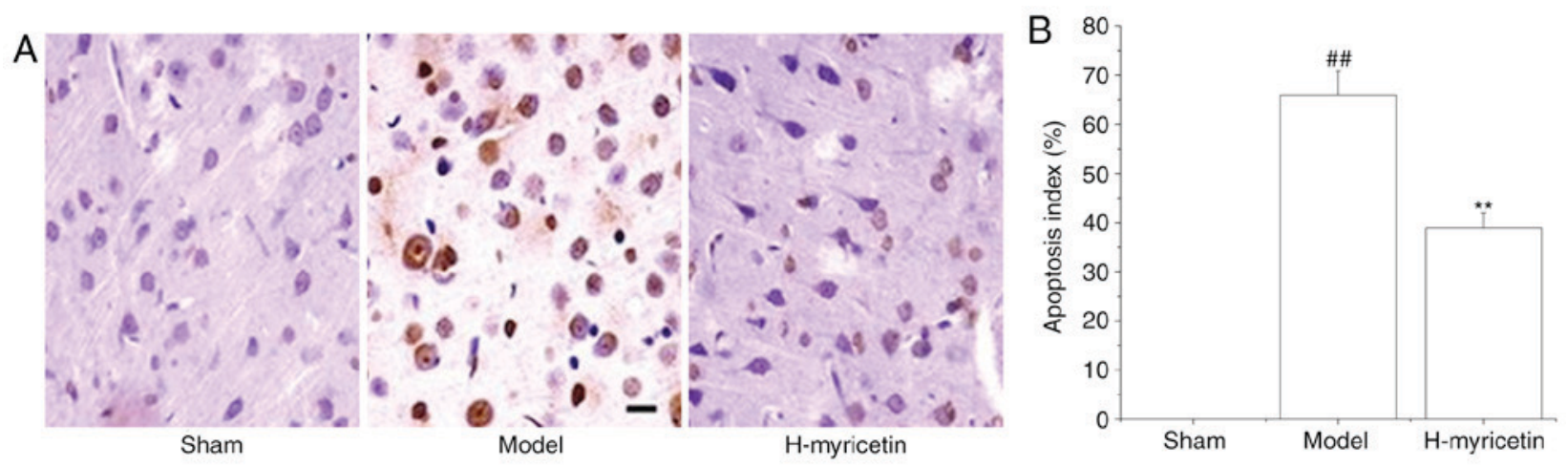

Figure 4. Myricetin preconditioning significantly inhibits neuronal apoptosis. (A) Suppression of neuronal apoptosis by myricetin (x400). Brown staining of the nucleus is indicative of apoptosis. (B) Percentage of apoptotic cells. Data are expressed as the mean \pm standard deviation ${ }^{\# \#} \mathrm{P}<0.01$ vs. Sham group; ${ }^{* *} \mathrm{P}<0.01$ vs. Model group, $\mathrm{n}=6$. H, high.
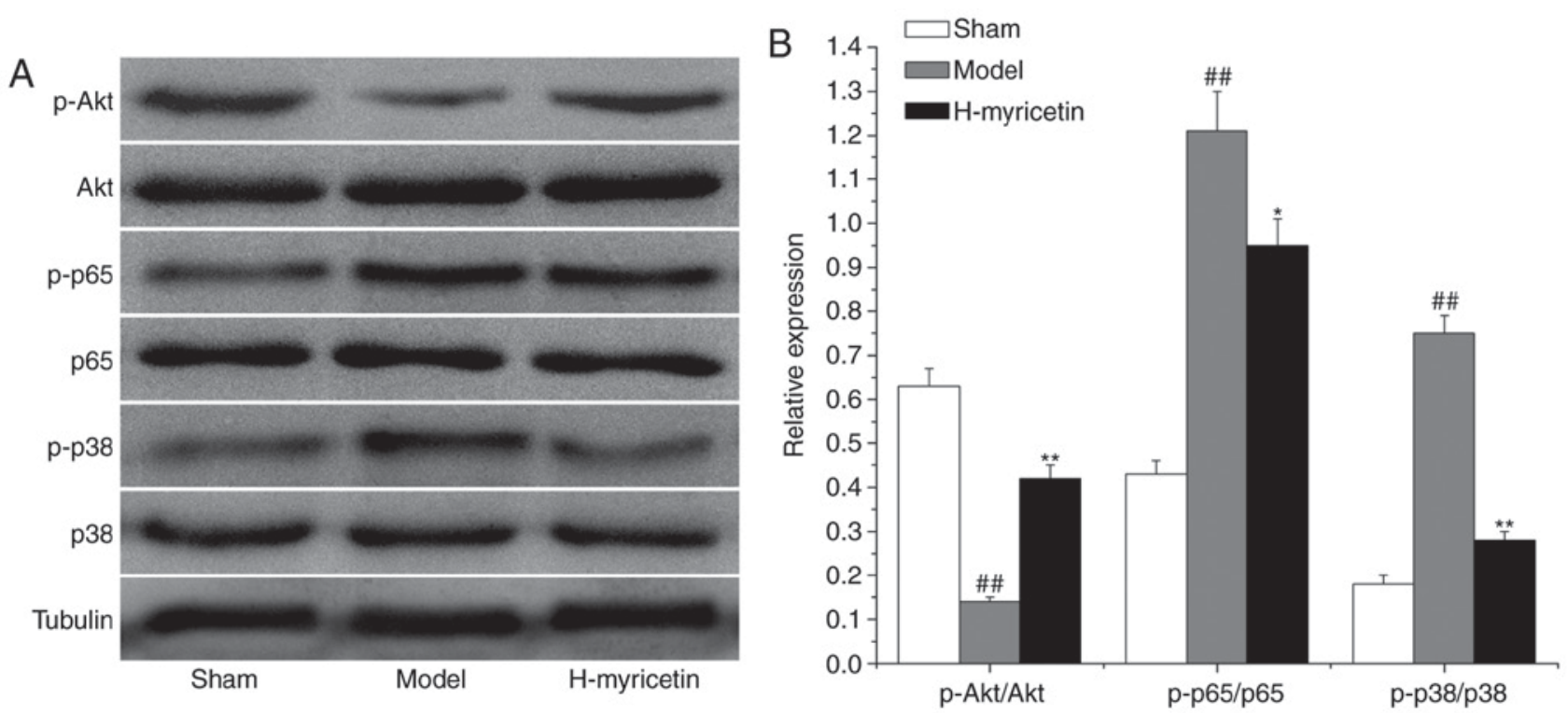

Figure 5. Effect of myricetin on the expression of proteins associated with apoptosis, inflammation and oxidative stress. (A) Expression of total and phosphorylated p38 MAPK, NF- $\mathrm{kB} / \mathrm{p} 65$ and AKT in brain tissue analyzed by western blotting. (B) Quantitative analysis of protein levels. Data are expressed as the mean \pm standard deviation ${ }^{\# \#} \mathrm{P}<0.01$ vs. Sham group; ${ }^{* *} \mathrm{P}<0.01$ vs. Model group, $\mathrm{n}=6$. H, high; $\mathrm{p}$, phosphorylated; $\mathrm{p} 38$, MAPK, $\mathrm{p} 38$ mitogen activated protein kinase; NF-kB/p65, nuclear factor; AKT, protein kinase B.

factors. Levels of inflammatory cytokines were significantly decreased in the $\mathrm{H}$-myricetin group $(\mathrm{P}<0.01)$ compared with the model group.

Myricetin suppresses apoptosis in ischemic brain tissue. As presented in Fig. 4, there was almost no apoptosis in the sham group. pMCAO induced an increase in apoptosis in the model group $(\mathrm{P}<0.01$, Apoptosis index: 65.94 \pm 4.89$)$. Compared with the model group, treatment with $\mathrm{H}$-myricetin substantially reduced the ischemia-induced neuronal apoptosis $(\mathrm{P}<0.01$, apoptosis index: $38.79 \pm 3.12$ ).

Effect of myricetin treatment on proteins associated with oxidative stress, inflammation and apoptosis in the ischemic brain tissue. To study the possible mechanisms of myricetin's neuroprotective effects, the expression of proteins associated with apoptosis, inflammation, and oxidative stress (AKT, $\mathrm{NF}-\kappa \mathrm{B} / \mathrm{p} 65$ and $\mathrm{p} 38 \mathrm{MAPK}$, respectively) were measured via western blot analysis. As presented in Fig. 5, compared with the sham group, a decreased level of phosphorylated AKT and increased levels of phosphorylated p38 MAPK and NF- $\kappa \mathrm{B} / \mathrm{p} 65$ were observed in the model group $(\mathrm{P}<0.01)$. Compared with the model group, treatment with $\mathrm{H}$-myricetin significantly increased the relative level of phosphorylated AKT and decreased the levels of phosphorylated p38 MAPK and NF- $\mathrm{kB} / \mathrm{p} 65$.

\section{Discussion}

The results of the present study demonstrated that myricetin treatment markedly attenuated ischemia-induced cerebral injury, considerably abated the ischemia-induced brain injury, and reduced inflammatory response, apoptosis, and oxidative stress in the brain tissue. The neuroprotective effect of myricetin may be associated with the activation of the AKT pathway and the inactivation of the NF- $\mathrm{kB} / \mathrm{p} 65$ and p38 MAPK pathways.

Ischemia-induced cerebral injury leads to cerebral infarction and, ultimately, brain dysfunction $(16,17)$. In the present 
study, it was observed that ischemia exacerbated neurological deficits and increased cerebral infarction. A previous study demonstrated that myricetin protects against ischemic cerebral injury via antioxidant properties (14). It was observed that treatment with myricetin attenuated cerebral infarction and the neurological deficits induced by ischemia. Additionally, treatment with myricetin significantly reduced the content of MDA (a critical component of reduced nicotinamide adenine dinucleotide phosphate oxidase), and reversed the ischemia-induced reduction of total antioxidant capacity (SOD activity), in addition to the GSH/GSSG ratio. Therefore, these findings suggest that myricetin decreased cerebral ischemia injury via the regulation of oxidative stress.

The inflammatory reaction is additionally involved in ischemia-induced cerebral injury (18). Inflammatory cell infiltration is an early step in the process of cerebral ischemic injury, and the activation of $\mathrm{NF}-\kappa \mathrm{B} / \mathrm{p} 65$ is a primary pathway that modulates cerebral inflammation $(19,20)$. Downregulation of the $\mathrm{NF}-\kappa \mathrm{B}$ pathway reduces the release of pro-inflammatory mediators (intercellular adhesion molecule-1, IL-1 $\beta$, IL-6, and IL-8), therefore reducing the cerebral ischemic injury in rats (21). A previous study revealed that myricetin exhibits a potent anti-inflammatory effect (22). The present study demonstrated that treatment with myricetin markedly inhibited the ischemia-induced increased levels of proinflammatory factors and activities of cytokines, including IL-1 $\beta$, TNF- $\alpha$, IL-6 and NF- $\kappa$ B/p-p65. The findings suggested that myricetin is a promising natural product that may be administered to counteract the ischemia-induced cerebral inflammatory response.

Cell apoptosis exhibits a key role in the development of cerebral infarction and brain dysfunction following ischemia (23). Consequently, inhibiting oxidative stress is considered a viable way of treating ischemia-induced cerebral injury. The results of the present study demonstrated that ischemia significantly increased neuronal apoptosis. It was observed that treatment with myricetin significantly reduced neuronal apoptosis. This finding suggested that myricetin exhibits a neuroprotective role, which may be via anti-apoptosis.

Various signaling pathways are associated with neuroprotective effects, including NF- $\kappa$, p38 MAPK and AKT $(24,25)$. AKT exhibits a key role in strengthening cell survival through the inhibition of caspase-activated apoptosis (26). Studies have demonstrated that increasing phosphorylation of AKT inhibits the activation of glycogen synthase kinase (GSK)-3 $\beta$, resulting in inhibition of caspase-activated apoptosis. A previous study additionally demonstrated that the AKT inhibitor (MK2206) suppresses the phosphorylation of AKT, followed by the activation of GSK-3 $\beta$, which increases apoptosis (27). p38 MAPK is also important in response to ischemic cerebral injury, and is involved in various physiological processes, specifically, oxidative stress injury, inflammatory response injury and neuronal apoptosis $(28,29)$. Various studies have demonstrated that inhibition of p38 MAPK reduces ischemia-induced cerebral injury $(30,31)$. The results of the present study indicated that treatment with myricetin following cerebral ischemic injury led to inhibition of p38 MAPK and activation of AKT signaling pathways. Therefore, myricetin may protect against ischemia-induced cerebral injury, possibly through the modulation of multiple signaling pathways.
In accordance with the results of a previous study (14), the present study demonstrated that myricetin reduced the oxidative stress in brain ischemia injury. Additionally, the present study revealed that myricetin reduced the inflammatory response in ischemic brain injury tissue. Furthermore, it was indicated that the effects of myricetin in decreasing cerebral ischemia injury may be associated with inhibition of p38 MAPK and NF- $\mathrm{B}$ pathway, and activation of AKT signaling pathways.

In conclusion, the results from the present study suggested that treatment with myricetin decreased the size of cerebral infarction, inhibited apoptosis, reduced inflammation and decreased oxidative stress. Additionally, it was observed that these effects may be associated with inhibition of p38 MAPK and activation of AKT pathways in the ischemia-injured brain.

\section{Acknowledgements}

The present study (grant no. 20150102516) was supported by Lin Yi Central Hospital.

\section{References}

1. Brill AK, Rösti R, Hefti JP, Bassetti C, Gugger M and Ott SR: Adaptive servo-ventilation as treatment of persistent central sleep apnea in post-acute ischemic stroke patients. Sleep Med 15: 1309-1313, 2014

2. Jovanovic A, Stolic RV, Rasic DV, Markovic-Jovanovic SR and Peric VM: Stroke and diabetic ketoacidosis-some diagnostic and therapeutic considerations. Vasc Health Risk Manag 10: 201-204, 2014.

3. Lozano R, Naghavi M, Foreman K, Lim S, Shibuya K, Aboyans V, Abraham J, Adair T, Aggarwal R, Ahn SY, et al: Global and regional mortality from 235 causes of death for 20 age groups in 1990 and 2010: A systematic analysis for the Global Burden of Disease Study 2010. Lancet 380: 2095-2128, 2012.

4. Murray CJ, Vos T, Lozano R, Naghavi M, Flaxman AD, Michaud C, Ezzati M, Shibuya K, Salomon JA, Abdalla S, et al: Disability-adjusted life years (DALYs) for 291 diseases and injuries in 21 regions, 1990-2010: A systematic analysis for the Global Burden of Disease Study 2010. Lancet 380: 2197-2223, 2012.

5. Writing Group Members, Mozaffarian D, Benjamin EJ, Go AS, Arnett DK, Blaha MJ, Cushman M, Das SR, de Ferranti S, Després JP, et al: Executive summary: Heart disease and stroke statistics-2016 update: A report from the American Heart Association. Circulation 133: 447-454, 2016.

6. Strong K, Mathers C and Bonita R: Preventing stroke: Saving lives around the world. Lancet Neurol 6: 182-187, 2007.

7. Tewari A, Mahendru V, Sinha A and Bilotta F: Antioxidants: The new frontier for translational research in cerebroprotection. J Anaesthesiol Clin Pharmacol 30: 160-171, 2014.

8. Liang LJ, Yang JM and Jin XC: Cocktail treatment, a promising strategy to treat acute cerebral ischemic stroke. Med Gas Res 6: 33-38, 2016.

9. Wang W, Ma X, Han J, Zhou M, Ren H, Pan Q, Zheng C and Zheng Q: Neuroprotective effect of scutellarin on ischemic cerebral injury by down-regulating the expression of angiotensin-converting enzyme and AT1 receptor. PLoS One 11: e0146197, 2016.

10. Kang KA, Wang ZH, Zhang R, Piao MJ, Kim KC, Kang SS, Kim YW, Lee J, Park D and Hyun JW: Myricetin protects cells against oxidative stress-induced apoptosis via regulation of PI3K/Akt and MAPK signaling pathways. Int J Mol Sci 11: 4348-4360, 2010

11. Majid M, Khan MR, Shah NA, UI Haq I, Farooq MA, Ullah S, Sharif A, Zahra Z, Younis T and Sajid M: Studies on phytochemical, antioxidant, anti-inflammatory and analgesic activities of Euphorbia dracunculoides. BMC Complement Altern Med 15: $349,2015$. 
12. Mendes V, Vilaça R, de Freitas V, Ferreira PM, Mateus N and Costa V: Effect of myricetin, pyrogallol, and phloroglucinol on yeast resistance to oxidative stress. Oxid Med Cell Longev 2015: 782504, 2015.

13. Qiu Y, Cong N, Liang M, Wang Y and Wang J: Systems pharmacology dissection of the protective effect of myricetin against acute ischemia/reperfusion-induced myocardial injury in isolated rat heart. Cardiovasc Toxicol 17: 277-286, 2017.

14. Wu S, Yue Y, Peng A, Zhang L, Xiang J, Cao X, Ding H and Yin S: Myricetin ameliorates brain injury and neurological deficits via Nrf2 activation after experimental stroke in middle-aged rats. Food Funct 7: 2624-2634, 2016.

15. Longa EZ, Weinstein PR, Carlson S and Cummins R: Reversible middle cerebral artery occlusion without craniectomy in rats. Stroke 20: 84-91, 1989.

16. Livnat A, Barbiro-Michaely E and Mayevsky A: Mitochondrial function and cerebral blood flow variable responses to middle cerebral artery occlusion. J Neurosci Methods 188: 76-82, 2010.

17. Ahn HC, Yoo KY, Hwang IK, Cho JH, Lee CH, Choi JH, Li H, Cho BR, Kim YM and Won MH: Ischemia-related changes in naive and mutant forms of ubiquitin and neuroprotective effects of ubiquitin in the hippocampus following experimental transient ischemic damage. Exp Neurol 220: 120-132, 2009.

18. Xiang Y, Zhao H, Wang J, Zhang L, Liu A and Chen Y: Inflammatory mechanisms involved in brain injury following cardiac arrest and cardiopulmonary resuscitation. Biomed Rep 5: 11-17, 2016.

19. Chen YF, Wang YW, Huang WS, Lee MM, Wood WG, Leung YM and Tsai HY: Trans-Cinnamaldehyde, An essential oil in cinnamon powder, ameliorates cerebral ischemia-induced brain injury via inhibition of neuroinflammation through attenuation of iNOS, COX-2 expression and NFk-B signaling pathway. Neuromolecular Med 18: 322-333, 2016

20. Wang YS, Li YX, Zhao P, Wang HB, Zhou R, Hao YJ, Wang J, Wang SJ, Du J, Ma L, et al: Anti-inflammation effects of oxysophoridine on cerebral ischemia-reperfusion injury in mice. Inflammation 38: 2259-2268, 2015.

21. Xu S, Zhong A, Ma H, Li D, Hu Y, Xu Y and Zhang J: Neuroprotective effect of salvianolic acid B against cerebral ischemic injury in rats via the $\mathrm{CD} 40 / \mathrm{NF}-\kappa \mathrm{B}$ pathway associated with suppression of platelets activation and neuroinflammation. Brain Res 1661: 37-48, 2017.
22. Chanput W, Krueyos N and Ritthiruangdej P: Anti-oxidative assays as markers for anti-inflammatory activity of flavonoids. Int Immunopharmacol 40: 170-175, 2016.

23. Chen YF, Wu KJ, Huang WS, Hsieh YW, Wang YW, Tsai HY and Lee MM: Neuroprotection of Gueichih-Fuling-Wan on cerebral ischemia/reperfusion injury in streptozotocin-induced hyperglycemic rats via the inhibition of the cellular apoptosis pathway and neuroinflammation. Biomedicine (Taipei) 6: 21, 2016.

24. Zhang S, Shao SY, Song XY, Xia CY, Yang YN, Zhang PC and Chen NH: Protective effects of Forsythia suspense extract with antioxidant and anti-inflammatory properties in a model of rotenone induced neurotoxicity. Neurotoxicology 52: 72-83, 2016

25. Zhu L, Bi W, Lu D, Zhang C, Shu X and Lu D: Luteolin inhibits SH-SY5Y cell apoptosis through suppression of the nuclear transcription factor- $\kappa \mathrm{B}$, mitogen-activated protein kinase and protein kinase B pathways in lipopolysaccharide-stimulated cocultured BV2 cells. Exp Ther Med 7: 1065-1070, 2014.

26. Fu P, Wu Q, Hu J, Li T and Gao F: Baclofen protects primary rat retinal ganglion cells from chemical hypoxia-induced apoptosis through the Akt and PERK pathways. Front Cell Neurosci 10: 255,2016

27. Ying Y, Zhu H, Liang Z, Ma X and Li S: GLP1 protects cardiomyocytes from palmitate-induced apoptosis via Akt/GSK3b/b-catenin pathway. J Mol Endocrinol 55: 245-262, 2015.

28. Qi Z, Qi S, Gui L, Shen L and Feng Z: Daphnetin protects oxidative stress-induced neuronal apoptosis via regulation of MAPK signaling and HSP70 expression. Oncol Lett 12: 1959-1964, 2016.

29. Suchal K, Malik S, Gamad N, Malhotra RK, Goyal SN, Chaudhary U, Bhatia J, Ojha S and Arya DS: Kaempferol attenuates myocardial ischemic injury via inhibition of MAPK signaling pathway in experimental model of myocardial ischemia-reperfusion injury. Oxid Med Cell Longev 2016: 7580731, 2016.

30. Apostolatos A, Song S, Acosta S, Peart M, Watson JE, Bickford P, Cooper DR and Patel NA: Insulin promotes neuronal survival via the alternatively spliced protein kinase CSII isoform. J Biol Chem 287: 9299-9310, 2012.

31. Cheng CY, Lin JG, Tang NY, Kao ST and Hsieh CL: Electroacupuncture at different frequencies $(5 \mathrm{~Hz}$ and $25 \mathrm{~Hz})$ ameliorates cerebral ischemia-reperfusion injury in rats: Possible involvement of p38 MAPK-mediated anti-apoptotic signaling pathways. BMC Complement Altern Med 15: 241, 2015. 